\title{
New Model for the Prediction of Vision-Related Outcomes in Young Children with Mechanical Ocular Conditions and Comparison with Other Models
}

Mehul A Shah ( $\nabla$ omtrustdahod@gmail.com )

Drashti Netralaya https://orcid.org/0000-0003-2811-3915

Shreya Shah

Drashti Netralaya

Apeksha Kataria

Drashti Netralaya

Ruhi Gunay

Drashti Netralaya

Sakshi Makhloga

Drashti Netralaya

Mrugesha Vaghela

Drashti Netralaya

\section{Research Article}

Keywords: OTS, POTS, TOTS, BETTS, TRAUMATIC CATARACT, PAEDIATRIC OCULAR TRAUMA

Posted Date: February 28th, 2022

DOI: https://doi.org/10.21203/rs.3.rs-986589/v1

License: (c) (i) This work is licensed under a Creative Commons Attribution 4.0 International License.

Read Full License 


\section{Abstract}

Purpose Herein, we compared the efficacy among the OTS, POTS, and TOTS for prognosis prediction in Indian children who had mechanical ocular conditions causing traumatic cataract.

Methods This prospective, interventional study recruited consecutive children undergoing operation for traumatic cataracts caused by mechanical eye injuries at Drashti Netralaya. The following details were obtained from medical files: the circumstance and time of injuries, penetrating injury type, initial and final visual acuity (VA), time of operation, and associated eye diseases. Specific variables were employed to determine the OTS, TOTS, and POTS. For all patients, the final and predicted VA determined using all scores were compared using Fischer's exact test; the accuracy, specificity, and sensitivity were evaluated for all scores by using the AUROC.

Results We enrolled 124 eyes. Patients' mean value for age was $4.6 \pm 1.29$ years; 44 (35.41\%) and 74 $(64.5 \%)$ were female and male patients, respectively. Visual outcomes significantly improved after operation, and the outcomes did not differ between closed- and open-globe injuries $(P=0.162)$. The actual and predicted VA did not exhibit a statistically significant difference among the three scores. The TOTS and POTS were more suitable for evaluating low-risk injuries, whereas the OTS could more efficiently examine high-risk cases.

Conclusion The TOTS and POTS were more accurate than the OTS in VA prediction after operation in toddlers with traumatic cataracts caused by mechanical globe injury. All the examined scores can be helpful in estimating VA following treatment.

\section{Key Message}

1.What was known? POTS is useful predictive model based on presenting vision

What is new in this paper?

1.TOTS is sensitive and specific predictive model based on clinical findings.

2 This model is accurate in case of lower risk trauma

\section{Synopsis}

This TOTS is calculated based on clinical findings and not on presenting vision and tested for sensitivity and specificity for prediction, compared with POTS and OTS

\section{Introduction}

Ocular trauma does not receive adequate attention in many areas globally owing to poor infrastructure, untrained human resources, and variable outcomes. General ophthalmologists are the first-line health 
workers treating this eye condition, they are not particularly trained. Thus, the first assessment is performed by a relatively untrained team (REF LAJO).

Paediatric ocular trauma might result in life-long visual disability, thus posing a burden to the healthcare system and society. ${ }^{[1]}$ In children, open-globe injury is regarded as the most severe eye trauma caused by the penetration of a sharp object. ${ }^{[2]}$ To optimise outcomes, careful evaluation and timely treatment are vital in open-globe injury. Precise visual prognosis is a key challenge in eye trauma treatment in paediatric cases. Parents frequently question physicians whether their children would become blind or can see again. Different scoring methods available for ocular trauma evaluation can help healthcare providers appropriately predict vision-related outcomes. The Ocular Trauma Score (OTS) is one such generic instrument used to evaluate outcomes. In this scoring methodology, visual acuity (VA) is estimated by subtracting raw points for five diagnostic findings from initial VA. ${ }^{[3]}$ However, the OTS demonstrated a low accuracy in children owing to its inability to obtain accurate VA; thus, instruments specifically for paediatric patients should be developed. ${ }^{[4-8]}$ The Paediatric Penetrating Ocular Trauma Score (POTS) involves the exclusion of the initially calculated VA and afferent pupil defect serving as prognostic factors and the inclusion of wound location and age as new variables. ${ }^{[8]}$ However, POTS's drawbacks have been reported. ${ }^{[9-13]}$

Ocular trauma can result in the development of cataracts ${ }^{1}$. Various methodologies can be employed to determine vision-related outcomes in cataracts caused by trauma or other factors. ${ }^{5}$ However, in those with traumatic cataracts, damage caused to adjacent ocular tissues can possibly reduce visual gain following surgery. This phenomenon can result in different rates of success between patients with and without traumatic cataracts. Moreover, the vision-related outcomes of paediatric traumatic cataract cases are often unsatisfactory owing to recurrent inflammation and amblyopia (5).

The Birmingham Eye Trauma Terminology System (BETTS) provides standardised definitions for ocular trauma (5). These definitions can be employed to compare vision-related outcomes after operation for traumatic cataract and identify determinants that can predict the aforementioned outcomes. Most studies investigating the vision-related outcomes of traumatic cataracts (7) have included a small sample size or were designed as case studies.

The Toddler/Infant OTS (TOTS) was recently designed for children aged $<6$ years with open-globe injury resulting from trauma. In line with the POTS, the TOTS does not rely on VA and was beneficial for prognosis prediction in US toddlers and infants. ${ }^{[14,15]}$

\section{Methods}

\section{Patients}

This prospective cohort study was approved by the Drashti Netralaya ethics committee. (RefDN/2020/13)All procedures followed the tenets of the Declaration of Helsinki and its amendments. 


\section{Sample And Data Acquisition}

We included all patients aged $<6$ years who had traumatic cataracts resulting from ocular trauma and presented at the Drashti Netralaya between January 2011 and December 2020. We excluded children with a follow-up duration of $<6$ months. We reviewed patients' electronic medical records. Data retrieved included demographic factors, ocular trauma characteristics and clinical data in the pretested online format, VA at the final follow-up, and existence of concomitant ocular disease. Information for the following additional signs and ocular comorbidities used to calculate scores was collected: hyphema, iris organic/unclean injury, prolapse, detachment of the retina, vitreous haemorrhage, traumatic cataract delay of $>48 \mathrm{~h}$ for surgery, and endophthalmitis.

\section{Score Calculations And Statistical Analyses}

We determined the OTS by using the standard table of raw points and points deducted according to comorbidities by converting them into different OTS categories.

Furthermore, the POTS was determined following the method reported by Acar et al and Shah et al..$^{\left[{ }^{-}--\right]}$ We assigned raw points to VA calculated at the presentation. Additional points were added or subtracted for wound location, age, and any of the eight concomitant eye conditions. For patients with missing information regarding initial VA in medical records, the following equation was adopted for trauma score calculation: $\mathrm{VA}=2 \times($ age + zone $)-$ corresponding pathologies.

We stratified open-globe injuries into three anatomical zones based on their location: zone I (those confined to the limbus and cornea), zone II (those located $5 \mathrm{~mm}$ posteriorly to the limbus), and zone III (if the wound was extended to the macula and optic nerve posteriorly to zone II). ${ }^{[15]}$ The range of the resulting score was from 1 (low prognosis) to 5 (favourable prognosis).

The following injury-related characteristics were evaluated to determine the TOTS: wound size $>6 \mathrm{~mm}$, existence of hyphema, cataract or lens damage, choroidal detachment, and detachment of the retina; these characteristics were assigned 1, 1, 1, 2, and 1 point, respectively, and zones II and III were assigned 1 and 2 points, respectively. ${ }^{[14]}$ We considered the injury as having low (favourable prognosis) and high (poor prognosis) risk if the sum of points was 0 or 1 and $\geq 2$, respectively. ${ }^{[14]}$

The OTS categories 1-5 and POTS 1-5 were calculated according to vision improvements. We compared the results of OTS categories 1-5 with those of TOTS categories 5-1 and those of POTS categories $1-5$ with those of TOTS categories $5-1$ by using Fisher's exact test and Student's $t$ test with $95 \%$ Cls. A P value of $<0.5$ was considered significant.

The ROC curves comparing the TOTS with the OTS and the POTS with the TOTS for all categories were plotted to examine their specificity and sensitivity. 
To perform statistical analysis, POTS and OTS categories 2-5 were all merged into the low-risk category corresponding to the TOTS category 1 . The scoring methods' predictive values were examined through a comparison of final and predicted VA by using Fisher's exact test of independence; the sample t test was performed to evaluate its association. Specificity and sensitivity with $95 \%$ Cls were calculated using the ROC curve for each score. A P value of $<0.05$ indicated significance.

\section{Results}

Of 1630 traumatic cataract cases included, $457(28 \%)$ and $1173(72 \%)$ were female and male patients, respectively. Furthermore, $681(41.8 \%)$ were paediatric cases.

We studied 124 patients aged < 6 years; among them, 44 (35.41\%) and 74 (64.5\%) were female and male patients, respectively (Table 1). Furthermore, the patients' mean and median ages were $4.6 \pm 1.29$ years and 5 years, respectively. Among 124 patients, 29 (23.4\%) and 95 (76.6\%) had closed-globe and openglobe injuries, respectively. The pre- and post-vision differed significantly. The outcomes of open-globe injury did not differ significantly from those of closed-globe injury $(P=0.162)$. Moreover, children aged $<2$ years had significantly poorer outcomes.

The number and percentage of cases with POTS and OTS categories 1-5 and TOTS categories 5-1 (more to less severe) were determined.

The difference among OTS, TOTS, and POTS categories was nonsignificant (Table 2)

No significant difference was noted between the low-risk TOTS categories and the merged 2-5 categories of POTS $(P=0.241$ and 0.241 , respectively; Table 2$)$.

Sensitivity and specificity for TOTS compared to OTS and POTS is displayed in Table-3 (for merged low risk categories) and Figure-2. (For individual categories)

\section{Discussion}

This study investigated the applicability and validity of TOTS, OTS, and POTS in patients aged from 0 to 6 years. The findings indicated the higher applicability of the OTS than the POTS in general population.

The OTS provides a more accurate prediction of vision-related outcomes. Nevertheless, the inclusion of children in a database of $>2500$ patients with severe eye injury based on which the score was formulated remains unclear $[5,6]$.

Although studies have employed regression tree, its validity is not evaluated. Compared with regression tree analysis, the OTS provided a highly accurate prognosis prediction $(8,9,10)$.

A study developed a predictive model for open-globe injuries (9). 
Another study developed the Basic Severity Score for Common Ocular Emergencies for examining the severity status of 86 common eye conditions by using the Delphi method. In this methodology, the severity was rated using a scale (7 point) in the first round of the survey. The final severity of each item was determined according to the median ratings obtained in the final Delphi survey. However, this score is not used widely and remains to be validated (11).

Politzer et al developed the Craig Hospital Eye Evaluation Rating Scale (CHEERS) to investigate the severity and frequency of deficits in eye movements in traumatic brain injury patients (12).

Lesniak and Bauza (13) found that in paediatrics patients, the final calculated VA did not significantly differ from the VA predicted with the OTS. Sharma suggested that the initially calculated OTS might predict the prognosis of paediatric penetrating eye injury patients (14). However, Unver (15) indicated the limitation of OTS in predicting vision-related outcomes in paediatric cases (15).

Studies have prospectively validated the OTS in children $(15,16)$.

Bunting, Schörkhuber, Hossain, Uysal Y, and Tok have indicated that the OTS exhibited high validity in paediatric open-globe injury cases (17-21).

The OTS could predict vision-related outcomes after operation in 354 traumatic cataract patients [22]. However, the likelihood of amblyopia should be considered in children. Refractive errors, strabismus, and ocular opacity can result in amblyopia. Not including amblyopia while calculating scores can reduce OTS's prediction accuracy. Therefore, we considered amblyopia in the OTS calculation for establishing a model for the POTS. We validated this model and compared prediction accuracy between the OTS and POTS for evaluating outcomes in paediatric traumatic cataract patients $(23,24)$.

A study conducted in India validated the OTS in 787 traumatic cataract individuals [19].

Because of difficulty in obtaining RAPD and initial VA, two crucial factors for OTS calculation, in paediatric trauma patients, particularly younger children, the calculated OTS would be inaccurate. Two studies conducted in Turkey calculated the OTS for children; however, these studies reported contradictory findings $[25,26]$. The POTS was recently developed for prognosis prediction in children whose initial vision findings were inaccurate [23].

In children, the POTS was more accurate than the OTS, as indicated by the AUROC (23).

The accuracy of POTS and OTS in examining the prognosis of open-globe injuries in paediatric cases was similar (28).

Zhu found that the POTS was more robust than the OTS in examining penetrating injuries (29).

The findings of regression analysis revealed that both OTS and CART were robust in predicting prognosis; however, the OTS demonstrated a greater accuracy. Thus, the OTS could be employed to counsel patients and make treatment-related decisions (8). 
This study examined the scoring methods' applicability and validity. The OTS but not the POTS demonstrated increased applicability in general population. Future studies should examine TOTS's applicability.

To our knowledge, no study has compared the validity among the TOTS, POTS, and OTS.

In line with the BETTS, the OTS could be easily used to evaluate both closed-globe and open-globe eye injury. The examination of six factors (A to $F$ ) for prediction required for OTS calculation is relatively easy. The OTS could accurately predict vision-related outcomes in open-globe injury. However, a 1-in-5 risk of obtaining an incorrect score exists; thus, this score should not be employed to plan the primary treatment procedure. The OTS should be adopted for making treatment-related decisions $(30,31)$. The TOTS and POTS exhibited limitations in those with poor prognosis.

Sarah et al developed a new prognostic score. Patients aged 0-6 years with open-globe injury exhibit specific risk factors indicating poor outcomes. The score calculated using our algorithm did not rely on VA acuity and thus can be beneficial for prognosis prediction in younger children (32).

Conclusion: TOTS, as a novel predictive score, is more reliable than the POTS but less specific and sensitive in high-risk (OTS-1 and POTS-1) cases. The TOTS can be employed to examine outcomes in toddlers in whom vision check-up is not possible on presentation.

\section{References}

1. Khatry SK, Lewis AE, Schein OD et al. The epidemiology of ocular trauma in rural Nepal. Br J Ophthalmol. 2004; 88:456-60.

2. Abraham, D. I.; Vitale, S. I.; West, S. I. et al. Epidemiology of eye injuries in rural Tanzania. Ophthalmic Epidemiol.1999; 6:85-94

3. Virgil Alfaro, Eric P. Jablon, Monica Rodriguez Fontal, Simon J. Villalba, Robert E.Morris, Michael Grossman, Enrique Roig-Melo et al. Fishing-related ocular trauma. American Journal of Ophthalmology.2005; 139: 488-492

4. Shah M, Shah S, Khandekar R. Ocular injuries and visual status before and after Their management in the tribal areas of Western India-A historical cohort study Grafes Arch Clin Exp Ophthalmol 2008; 246:191-197.

5. Kuhn F, Morris R, Witherspoon CD et al. The Birmingham Eye Trauma Terminology system (BETT). J Fr Ophtalmol. 2004; 27:206-10.

6. Kuhn, F., R. Maisiak, et al. "The Ocular Trauma Score (OTS)." Ophthalmol Clin North Am 2002;15(2): 163-5, vi.

7. Mehul A. Shah, Shreya M. Shah, Ashit H. Shah, Jaimini S. Pandya Visual outcome of cataract in pediatric age group: does aetiology have a role?Eur J Ophthalmol ( $2013 ;$ : 00) 000-00000 
8. Man, C. Y. and D. Steel "Visual outcome after open globe injury: a comparison of two prognostic models-the Ocular Trauma Score and the Classification and Regression Tree." Eye (Lond) 24(1): 849.

9. Schmidt G, Broman A, Grant M. Vision survival after open globe injury predicted by classification and regression tree analysis. Ophthalmology 2007; 115:202-9.

10. Yu Wai Man, C. and D. Steel "Visual outcome after open globe injury: a comparison of two prognostic models-the Ocular Trauma Score and the Classification and Regression Tree." Eye (Lond) 24(1): 849.

11. Bourges JL(1), Boutron I(2), Monnet D(3), Brézin AP(3).Consensus on Severity for Ocular Emergency: The BAsic SEverity Score for Common OculaR Emergencies [BaSe SCOrE].

12. Politzer T(1), Berryman A(2), Rasavage K(3), Snell L(4), Weintraub A(5), GerberDJ(6). The Craig Hospital Eye Evaluation Rating Scale (CHEERS). PM R. 2017 May; 9(5):477-482. Doi: 10.1016/j.pmrj.2016.08.032. Epub 2016 Sep 21.

13. Lesniak, S. P., A. Bauza, "Twelve-Year Review of Pediatric Traumatic Open Globe Injuries in an Urban U.S. Population." J Pediatr Ophthalmol Strabismus: 1-7.

14. Sharma, H. E., N. Sharma, et al. "Comment on a new ocular trauma score in pediatric penetrating eye injuries." Eye (Lond) 25(9): 1240.

15. Unver, Y. B., N. Acar, et al. (2008). "Visual predictive value of the ocular trauma score in children." $\mathrm{Br} \mathrm{J}$ Ophthalmol 2008;92(8): 1122-4.

16. Oiticica-Barbosa MM (1), Kasahara N (2) Eye trauma in children and adolescents: Perspectives from a developing country and validation of the ocular trauma score. J Trop Pediatr. 2015 Aug; 61(4):23843. Doi: 10.1093/tropej/fmv010. Epub 2015 Mar 29

17. Uysal Y (1), Mutlu FM, Sobaci G. Ocular Trauma Score in childhood open-globe injuries. J Trauma. 2008 Dec; 65(6):1284-6. Doi: 10.1097/TA.0b013e31817de3cc.

18. Tok O(1), Tok L, Ozkaya D, Eraslan E, Ornek F, Bardak Y. Epidemiological characteristics and visual outcome after open globe injuries in children.J AAPOS. 2011.Dec;15(6):55661.doi:10.1016/j.jaapos.2011.06.012

19. Shah, M. A., S. M. Shah, S.B.Shah et al. Ocular Trauma Score: a useful predictor of visual outcome at six weeks in patients with traumatic cataract. Ophthalmology 2012;119(7): 1336-41

20. Morgan AM (1), Kasahara N (1) (2). Comparative Evaluation of the Prognostic Value Between the Ocular Trauma Score and the Pediatric Penetrating Ocular Trauma Score.J Craniofac Surg. 2018 Oct;29(7):1776-1779. Doi: 10.1097/SCS.0000000000004937.

21. Zhu L(1), Wu Z(1), Dong F(1), Feng J(2), Lou D(3), Du C(1), Ren P(1). Two kinds of ocular trauma score for pediatric traumatic cataract in penetrating eye injuries. Injury. 2015 Sep; 46(9):1828-33. Doi: 10.1016/j.injury.2015.04.024. Epub 2015 Apr 23.

22. Shah, M. A., S. M. Shah, S.B.Shah, et al. Ocular Trauma Score as a predictor of final visual outcomes in traumatic cataract cases in pediatric patients. J Cataract Refract Surg 2012; 38(6): 959-65. 
23. Shah $M A^{1}$, Agrawal $R^{2}$, Teoh $R^{3}$, Shah $S^{4}{ }^{4}$, Patel $K^{4}$, Gupta $S^{4}$, Gosai $S^{4}$.Graefes Arch Clin Exp Ophthalmol.2017 May; 255(5):1027-1036. Doi: 10.1007/s00417-017-3616-y. Epub

.Pediatric ocular trauma score as a prognostic tool in the management of pediatric traumatic cataracts.

24. Acar U (1), Tok OY, Acar DE, Burcu A, Ornek F. A new ocular trauma score in pediatric penetrating eye injuries. Eye (Lond). 2011 Mar; 25(3):370-4. Doi: 10.1038/eye.2010.211. Epub 2011 Jan 21.

25. Lima-Gomez V., Blanco-Hernandez D M (2010) Ocular trauma scores at the initial evaluation of ocular trauma. Cir Cir.; 78:209-13.

26. Knyazer B, Levy J Prognostic factors in posterior open globe injuries (zone-III injuries). Clin Experiment Ophthalmol; 2008;36: 836-41.

27. Mao CJ (1), Yan H. Clinical characteristics of mechanical ocular injury and application of ocular trauma score].Zhonghua Yan Ke Za Zhi. 2012 May; 48(5):432-5.

28. Morgan AM (1), Kasahara N (1) (2). Comparative Evaluation of the Prognostic Value Between the Ocular Trauma Score and the Pediatric Penetrating Ocular Trauma Score Craniofac Surg. 2018 Oct;29(7):1776-1779. Doi: 10.1097/SCS.0000000000004937.

29. Zhu L(1), Wu Z(1), Dong F(1), Feng J(2), Lou D(3), Du C(1), Ren P(1). Two kinds of ocular trauma score for pediatric traumatic cataract in penetrating eye injuries. Injury. 2015 Sep; 46(9):1828-33. Doi: 10.1016/j.injury.2015.04.024. Epub 2015 Apr 23.

30. Robert scott the ocular trauma scott community eye health journal | volume 28 issue 91 | 2015

31. Shah M, Sundar G, Shah S. Ocular Trauma Score revisited - Making sense of it all. Lat Am J Ophthalmol 2019;2(4):1-8.

32. Read SP, Cavuoto KM. Traumatic open globe injury in young pediatric patients: characterization of a novel prognostic score. J AAPOS 2016; 20:141-144

\section{Tables}

Table-1 Age and sex distribution

\begin{tabular}{|lccc|}
\hline $\begin{array}{l}\text { AGE GROUP } \\
\text { IN YEARS }\end{array}$ & SEX & & TOTAL \\
& F & M & \\
$0-2$ & 4 & 5 & 9 \\
\hline $3-6$ & 40 & 75 & 115 \\
TOTAL & 44 & 80 & 124 \\
\hline
\end{tabular}

Table-2 Comparative study of OTS,POTS and TOTS for various trauma score categories 


\begin{tabular}{|lll|}
\hline VARIABLE1 & VARIABLE2 & P VALUE \\
\hline OTS1 & TOTS5 & NA \\
\hline POTS1 & TOTS5 & NA \\
\hline OTS2 & TOTS4 & 0.035 \\
\hline POTS2 & TOTS4 & 0.374 \\
\hline OTS3 & TOTS3 & 0.242 \\
\hline POTS3 & TOTS3 & 0.242 \\
\hline OTS4 & TOTS2 & 0.090 \\
\hline POTS4 & TOTS2 & 0.090 \\
\hline OTS5 & TOTS1 & 0.065 \\
\hline POTS5 & TOTS1 & 0.065 \\
\hline
\end{tabular}

Table-3 Study of sensitivity and specificity of different trauma categories (Area under curve)

\begin{tabular}{|c|c|c|c|}
\hline \multirow[t]{2}{*}{ TEST CATEGORY } & \multicolumn{2}{|c|}{ AREA UNDR CURVE } & \multirow[t]{2}{*}{ INFERENCE } \\
\hline & $\begin{array}{l}\text { FOR } \\
\text { OTS }\end{array}$ & $\begin{array}{l}\text { FOR } \\
\text { POTS }\end{array}$ & \\
\hline $\begin{array}{l}\text { ROC OTS POTS HIGHER RISK VS TOTS } \\
\text { HIGHER RISK }\end{array}$ & 000 & 000 & TOTS IS LESS RELAVENT \\
\hline $\begin{array}{l}\text { ROC OTS POTS LOWER RISK VS TOTS } \\
\text { LOWER RISK }\end{array}$ & 250 & 500 & $\begin{array}{l}\text { MORE SENTIVE AND } \\
\text { SPECIFIC }\end{array}$ \\
\hline ROC OTS 2 POTS2 VS TOTS 4 & 250 & 750 & $\begin{array}{l}\text { MORE SENSITIVE AND } \\
\text { SPECIFIC }\end{array}$ \\
\hline ROC OTS3 POTS3 VS TOTS3 & 833 & 250 & $\begin{array}{l}\text { MORE SENSITIVE AND } \\
\text { SPECIFIC }\end{array}$ \\
\hline ROC OTS 5 POTS5 VS TOTS1 & 500 & 500 & $\begin{array}{l}\text { LESS SENSITIVE AND } \\
\text { SPECIFIC }\end{array}$ \\
\hline
\end{tabular}

\section{Figures}



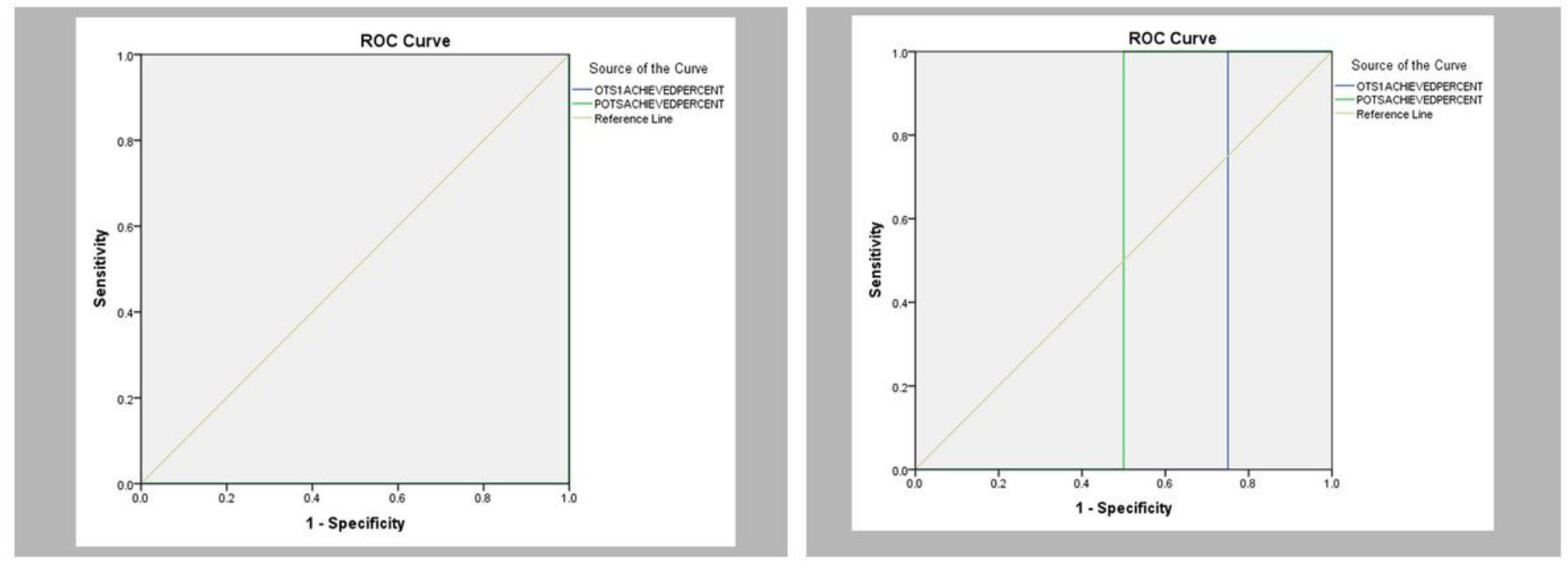

Figure 1

Caption not included with this version.
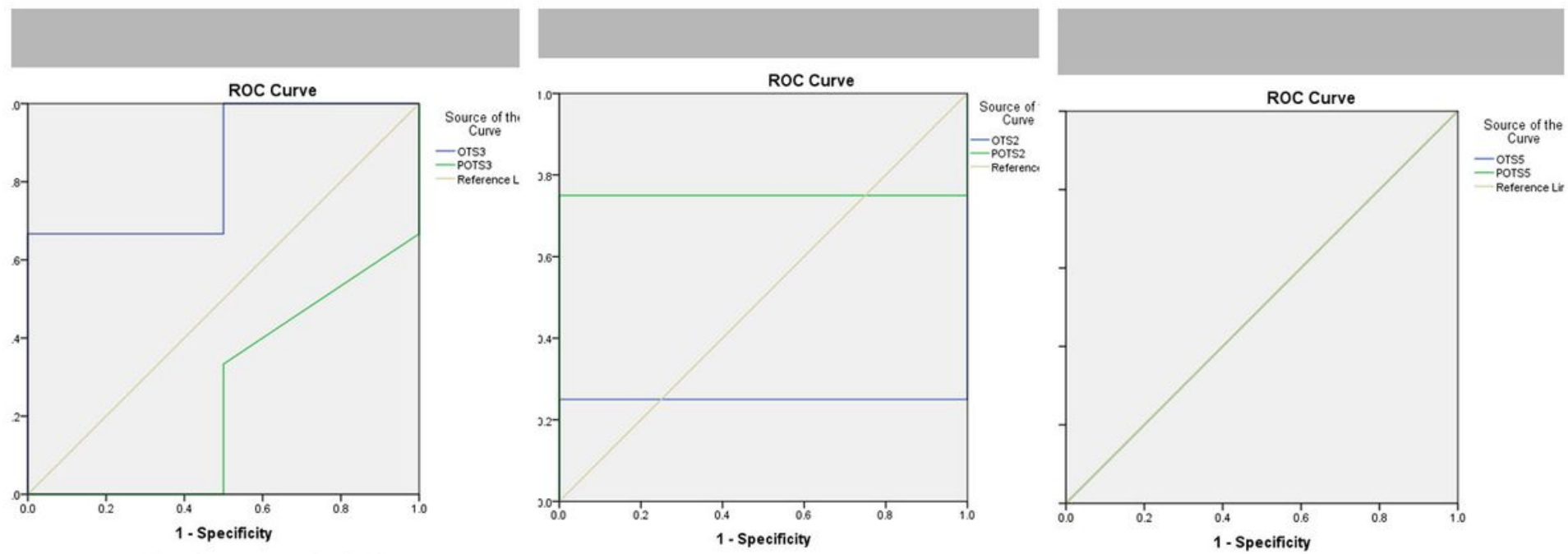

Figure 2

Caption not included with this version. 